\title{
CONTROL HÍBRIDO CONMUTADO COMO ALTERNATIVA TECNOLÓGICA PARA LA EFICIENCIA ENERGÉTICA
}

\author{
Julio A. Flórez-Vargas ${ }^{1}$, Ricardo Alzate-Castaño ${ }^{2}$
}

\author{
${ }^{1}$ Estudiante de la Maestría en Ingeniería Electrónica \\ 2 Profesor asistente. Correo electrónico: ralzatec@uis.edu.co \\ Escuela de Ingenierías Eléctrica, Electrónica y de Telecomunicaciones, Universidad Industrial de Santander, Bucaramanga
}

Recibido: 5 de agosto del 2013. Aprobado: 10 de octubre del 2013.

Cómo citar este artículo: J. A. Flórez-Vargas y R. Alzate-Castaño, "Control híbrido conmutado como alternativa tecnológica para la eficiencia energética". Ingeniería Solidaria, Vol. 9, No. 16, pp. 81-87, Dic., 2013.

Resumen. El presente artículo presenta técnicas de control híbrido como solución potencial al problema de regulación de los niveles de tensión a la salida de circuitos convertidores de potencia, para el mejoramiento de su eficiencia energética. Los sistemas de ejemplo corresponden con un convertidor DC-DC tipo Buck y un convertidor AC-DC tipo rectificador controlado basado en SCR. Se presenta una metodología para implementación de algoritmos de control híbrido dividida en tres pasos: modelado matemático, análisis numérico e implementación física. Se consideran como técnicas de ejemplo el control por modos deslizantes sMc y el control óptimo conmutado. Los mejores resultados en la práctica se obtienen con la técnica SMC. Los trabajos complementarios incluyen la revisión de aspectos prácticos para mejorar el desempeño del control óptimo y la aplicación de la metodología presentada en niveles de potencia mayores que permitan tener un impacto a escala industrial.

Palabras clave: control deslizante, control óptimo, convertidor de potencia, FPAA, FPGA, sistemas dinámicos híbridos.

\section{Switched Hybrid Control as an Alternative ENERgy EFficiency TechNology}

\begin{abstract}
This article presents hybrid control techniques as a potential solution to the problem of regulating output voltage levels from power conversion circuits to improve their energy efficiency. The example systems correspond to a DC-DC Buck converter and an AC-DC Silicon Controlled Rectifier (SCR) converter. A methodology is presented for implementing hybrid control algorithms in three steps: mathematical modeling, numerical analysis, and physical implementation. Sliding Mode Control (sMC) and optimal switched control are the example techniques considered. The best results in practice were obtained with the SMC technique. Complementary work included the review of practical aspects to improve the performance of optimal control and the application of the methodology at greater power levels that produce effects on an industrial scale.
\end{abstract}

Keywords: sliding control, optimal control, power converter, FPAA, FPGA, hybrid dynamic systems.

\section{CONTROLE HÍBRIDO COMUTADO COMO ALTERNATIVA TECNOLÓGICA PARA A EFICIÊNCIA ENERGÉTICA}

Resumo. O presente artigo apresenta técnicas de controle híbrido como solução potencial para o problema de regulação dos níveis de tensão na saída de circuitos convertedores de potência, para o melhoramento de sua eficiência energética. Os sistemas de exemplo correspondem com um conversor DC-DC tipo Buck e um conversor AC-DC tipo retificador controlado baseado em SCR. Apresenta-se uma metodologia para implementação de algoritmos de controle híbrido dividida em três passos: modelado matemático, análise numérica e implementação física. Consideram-se como técnicas de exemplo o controle por modos deslizantes sMC e o controle ótimo comutado. Os melhores resultados na prática se obtêm com a técnica SMc. Os trabalhos complementares incluem a revisão de aspectos práticos para melhorar o desempenho do controle ótimo e a aplicação da metodologia apresentada em níveis de potência maiores que permitam ter um impacto na escala industrial.

Palavras-chave: controle deslizante, controle ótimo, conversor de potência, FPAA, FPGA, sistemas dinâmicos híbridos. 


\section{Introducción}

Actualmente cobra vital importancia el buen uso que la humanidad pueda dar a los recursos energéticos. Fenómenos como el calentamiento global y la reducción de reservas naturales nos hacen pensar en la necesidad de administrar mejor la demanda de recursos por parte de una población en aumento y con una alta dependencia de la electricidad para satisfacer sus necesidades cotidianas. A nivel tecnológico, se reconoce al convertidor de potencia como el sistema que posee la responsabilidad de administrar de forma adecuada la transferencia energética entre una fuente y la carga que luego realizará el trabajo. Esta administración energética se traduce en acciones de regulación tradicionalmente resueltas mediante variaciones en la conmutación de los dispositivos semiconductores de los cuales está compuesto. En particular se emplea la variación del ciclo útil para una señal modulada en ancho de pulso (PWM). Soluciones convencionales, basadas por ejemplo en controladores PID [1] para modificar las condiciones de operación de un circuito convertidor de potencia, demuestran no ser las mejores como respuesta ante no linealidades del sistema o ante cambios paramétricos.

Como alternativa se plantean descripciones a manera de combinación entre formulaciones continuas y reglas discretas para el estudio de la conmutación en sistemas como el convertidor de potencia, a partir de los denominados sistemas dinámicos híbridos $[2,3]$. Este cambio de paradigma en la manera de analizar y manipular a través de acciones de control híbrido el comportamiento del circuito convertidor de potencia ha permitido obtener resultados importantes reportados en la literatura [2-6]. Algunos enfoques de control híbrido incluyen el control por modos deslizantes (SMC por sus siglas en inglés) [7] y el control óptimo [8], cuya formulación matemática busca la estabilidad del sistema a través de la teoría de estabilidad de Lyapunov [9] en complemento con la matemática hamiltoniana [10] y lagrangiana [11]. Por otro lado, las herramientas tecnológicas actuales facilitan la implementación de algoritmos complejos de control a partir del uso de dispositivos programables como los FPGA (del inglés, Field Programmable Gate Array) [3], los FPAA (del inglés, Field Programmable Analog Array) [5], los microcontroladores dsPIC [12], los PSoC (del inglés, Programmable System on-Chip) [13] o los DSP (del inglés, Digital Signal Processor) [4], combinando altas prestaciones de memoria y velocidad de procesamiento, con la flexi- bilidad dada por reconfiguraciones circuitales programadas. El presente artículo incluye resultados que apuntan en esta dirección, incorporando herramientas tecnológicas basadas en el diseño e implementación de controladores híbridos, para manipular el desempeño de circuitos convertidores de potencia $[6,14]$ como solución al problema de mantener regulados los niveles de tensión a la salida del circuito. El artículo se desarrollará en cuatro partes esenciales: la segunda sección abordará la metodología empleada para realizar la implementación de técnicas de control híbrido de tipo SMC y óptimo; la tercera, mostrará los resultados de laboratorio obtenidos tras la realización computacional de las rutinas de control sobre el circuito convertidor de potencia; en la cuarta se realizará una discusión que finalmente permitirá enunciar las conclusiones generales del trabajo en la quinta sección.

\section{Metodología}

La representación dinámica general de un sistema en el espacio de estados está dada por la expresión (1), en la que $x$ representa los estados, $u$ las entradas e $y$ las salidas del sistema $[7,9]$.

$$
\begin{aligned}
& \dot{x}=f(x, u) \\
& \dot{y}=g(x, u)
\end{aligned}
$$

Sin embargo, en la búsqueda por obtener un máximo aprovechamiento de energía se plantea la combinación de dinámicas en tiempo continuo con reglas de tiempo discreto, a partir de lo que se conoce como un sistema dinámico híbrido [3, 4]. En general, el esquema híbrido es aplicable a múltiples ramas del conocimiento que abarcan campos como la biología y la ingeniería. En el ámbito energético con enfoque en la obtención de recursos de fuentes no convencionales, se hace necesario mejorar el desempeño de la etapa de conversión de potencia $y$, de manera más precisa, disminuir en ella la mayor cantidad de pérdidas. Este aspecto impone la diferencia entre un sistema viable y otro inadecuado como una medida de costo-beneficio. No obstante, con la incorporación de tecnologías de implementación para acciones de control por medio de dispositivos programables, se facilita el avance en la búsqueda de una máxima eficiencia energética, convirtiendo a los convertidores de potencia en una clase de sistemas híbridos con mayor velocidad de procesamiento, ductilidad y portabilidad que sus predecesores no embebidos. 


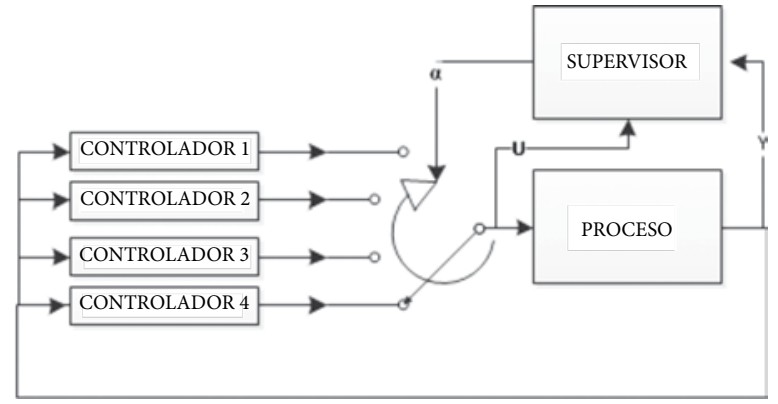

Figura 1. Control híbrido

Fuente: los autores

En la figura 1 se muestra un esquema genérico de un sistema dinámico híbrido. Para nuestro caso particular el proceso corresponde con el circuito convertidor de potencia. A su vez, el sistema supervisor se refiere a la tecnología de hardware programable capaz de realizar la conmutación con base en un algoritmo de control previamente implementado. La estructura metodológica desarrollada para llevar a cabo la implementación de un control híbrido conmutado en un convertidor de potencia se puede dividir en las etapas ilustradas en la figura 2 , enunciadas a continuación:

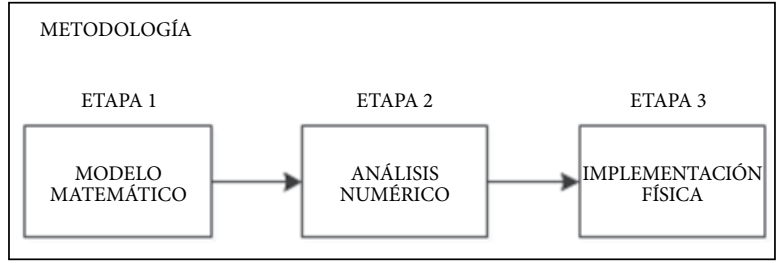

Figura 2. Planteamiento de metodología

Fuente: los autores

\subsection{Definición de la ley de control híbrido}

Como técnicas de control para verificar, se seleccionaron el smc y el control óptimo conmutado. Cada una de estas se formula a partir de las ecuaciones dinámicas del sistema. Ambas técnicas representan realizaciones binarias o digitales debido a la conmutación que constituyen como acción en el circuito convertidor de potencia, restringiendo sus valores de salida en el intervalo $(0,1)$.

El SMC como caso particular de control robusto tiene como característica principal la posibilidad de trabajar la estabilidad de un sistema vectorial n-dimensional como un sistema escalar, a través de una trayectoria representante del error conceptualizada como superficie de deslizamiento [7]. En (2) se puede ver la representación matemática de la superficie de deslizamiento, donde $x_{d}$ denota la trayectoria deseada - para nuestro caso el voltaje deseado a la salida del circui- to convertidor de potencia-, y en (3) la ley de control conmutado generada a partir del SMC, como una lectura del signo del error dinámico obtenido en camino hacia la superficie de deslizamiento.

$$
\begin{gathered}
s(t)=x(t)-x_{d}(t) \\
u(t)=\frac{1}{2}(1-\operatorname{sgn}(s))
\end{gathered}
$$

Por otra parte, el control óptimo tiene sus orígenes en la formulación matemática hamiltoniana, en donde la elección de la ley de control óptima se toma con base en su capacidad de minimizar un funcional de costo de energía $J$, típicamente asociado al gasto energético del actuador para lograr el óptimo desempeño del sistema. En (4) se muestra el funcional de costo obtenido para elegir la ley de control óptima para mantener constante la salida de un convertidor de potencia tipo Buck, como se puede observar en [15].

$$
J=\int_{0}^{T}\left(x(t)-x_{d}(t)\right)^{2}
$$

Esta elección se realiza según el principio del máximo del Pontryagin [16], donde la minimización de $J$ es equivalente a maximizar el hamiltoniano [10].

\subsection{Análisis numérico del sistema de control}

Una vez definida la ley de control, se realiza un análisis del comportamiento dinámico de los modelos matemáticos del sistema empleando herramientas computacionales, para predecir con respecto a la simulación posibles entornos virtuales de operación previos a la implementación física y definitiva del sistema. En particular, las pruebas realizadas en simulación buscan evidenciar la validez de las técnicas de control propuestas. Para el caso de análisis numérico en circuitos eléctricos y electrónicos, se dispone de herramientas de cálculo computacional como PSIM, Orcad y Proteus. Otro recurso importante y quizás más genérico está constituido por el paquete comercial MATLAB, el cual facilita la utilización de funciones matemáticas predefinidas para construir algoritmos genéricos. En la figura 3 se muestra un ejemplo de la gráfica de respuesta en el plano de fase [9] (i.e. voltaje vs. corriente) para un circuito convertidor de potencia tipo Buck, bajo la acción de un control por modos deslizantes simulada en matlab. En este caso la superficie de deslizamiento se eligió para mantener la salida de tensión constante en 7 
voltios. El eje $\mathrm{x}$ de la figura representa la corriente en la bobina y el eje y la tensión en la resistencia de carga. A partir de lo anterior se verifica la capacidad del sistema controlado para regresar a la superficie de deslizamiento posterior al efecto de una perturbación aplicada sobre la carga del sistema, como se verifica a partir de los dos puntos de acumulación de la trayectoria en la figura.

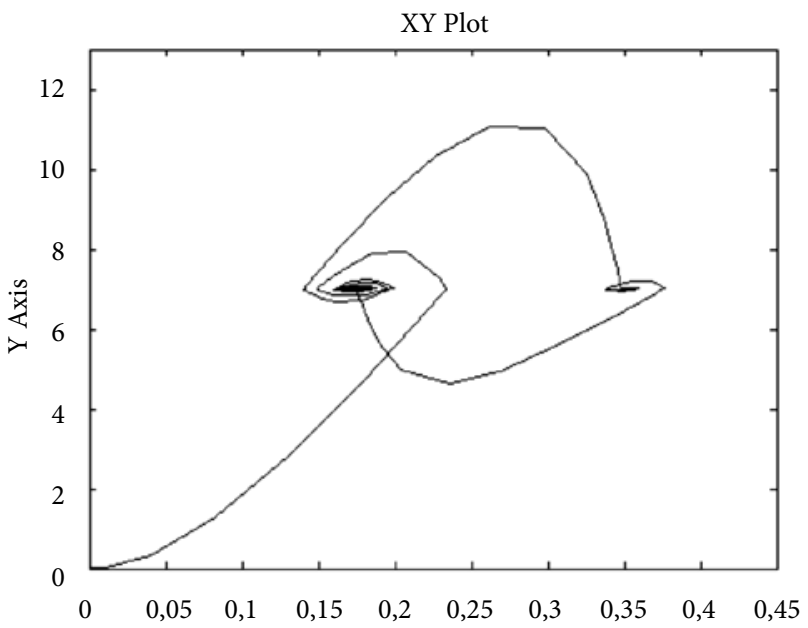

Figura 3. Plano de fase: Tensión (eje y $[\mathrm{V}]$ ) vs. Corriente (eje $\mathrm{x}$ [A]), para control deslizante

Fuente: los autores

\subsection{Selección para la tecnología de implementación}

Posterior a tener definida una ley de control híbrido para manipular la conmutación del circuito convertidor de potencia y verificar computacionalmente las predicciones teóricas de la dinámica controlada del mismo a través de simulación, se da paso a la siguiente etapa en la metodología propuesta para la selección de una alternativa adecuada para facilitar la realización circuital del algoritmo de control. En el presente artículo se consideran como opciones tecnológicas de implementación plataformas embebidas basadas en tecnologías FPAA y FPGA. En la figura 4 se puede observar la interfaz de programación gráfica ANADIGM-Designer ${ }^{\circledR}$ para el ANADIGM ${ }^{\circledR}$ AN221K04 basado en el FPAA AN120EO4.

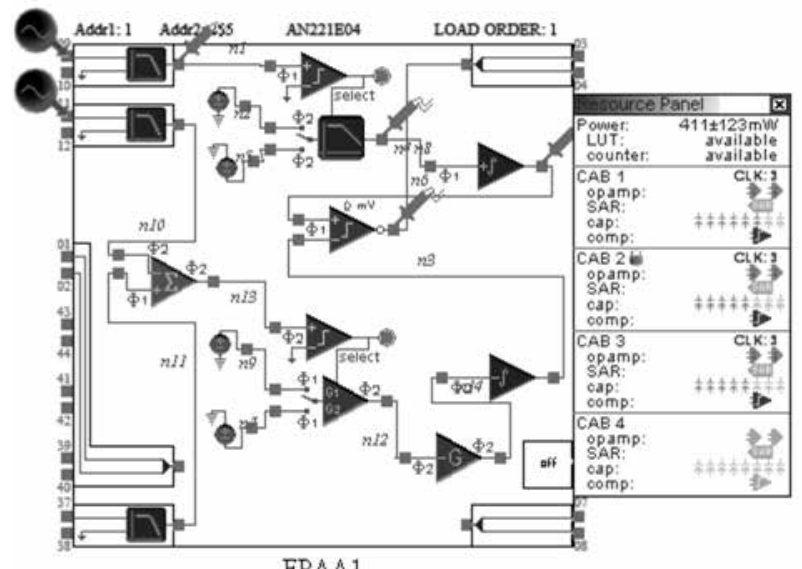

Figura 4. Entorno ANADIGM-Designer Fuente: los autores

De manera similar, el entorno de desarrollo integrado XILINX ISE ${ }^{\circledast}$ permite la programación en código VHDL para el sistema de desarrollo DIGILENT-NEXYs 3 basado en el FPGA Xilinx Spartan 6.

\section{Resultados}

A continuación se presentan los resultados obtenidos en laboratorio, para verificar el efecto de las acciones de control híbrido sobre el desempeño de circuitos convertidores de potencia. La figura 5 ilustra el diagrama de bloques del sistema experimental configurado, en el cual el dispositivo de proceso corresponde a alguna de las dos opciones: FPGA o FPAA. También es evidente que la señal de actuación corresponde con un bloque de PWM.

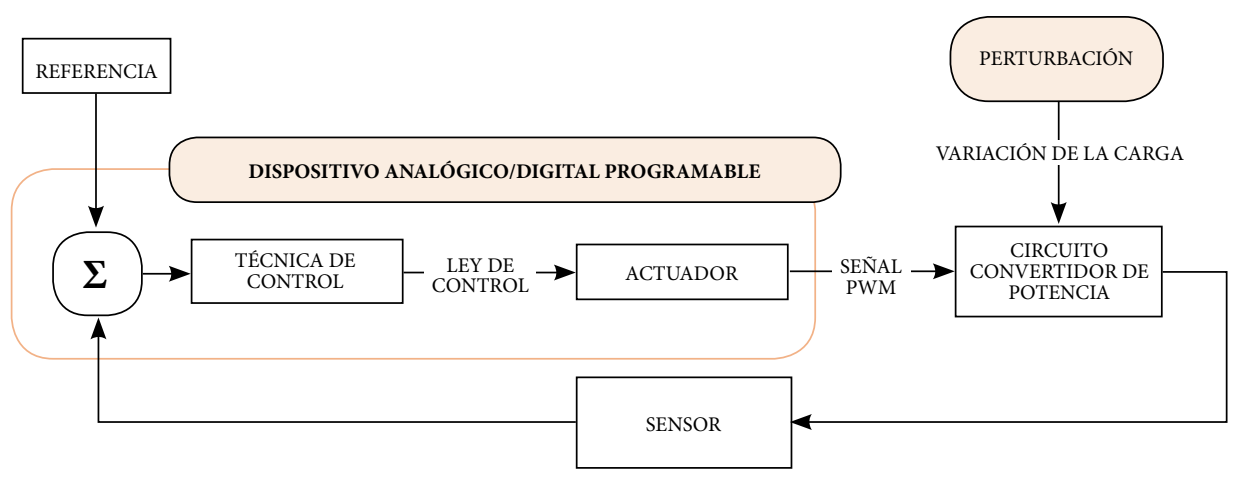

Figura 5. Esquema de control por entidades de decisión Fuente: los autores 
En la figura 6 se observa la señal de voltaje capturada a la salida de un convertidor DC-DC tipo Buck, sometido a perturbaciones en la salida, bajo la acción de un control por modos deslizantes implementado en un FPGA. De la figura se observa una respuesta plana, en la que rápidamente se corrige el efecto de la alteración aplicada en la impedancia de salida del sistema. Se nota también en la respuesta el rizado correspondiente a las micro-oscilaciones de alta frecuencia (chattering), generadas por la conmutación de los dispositivos semiconductores en el circuito.

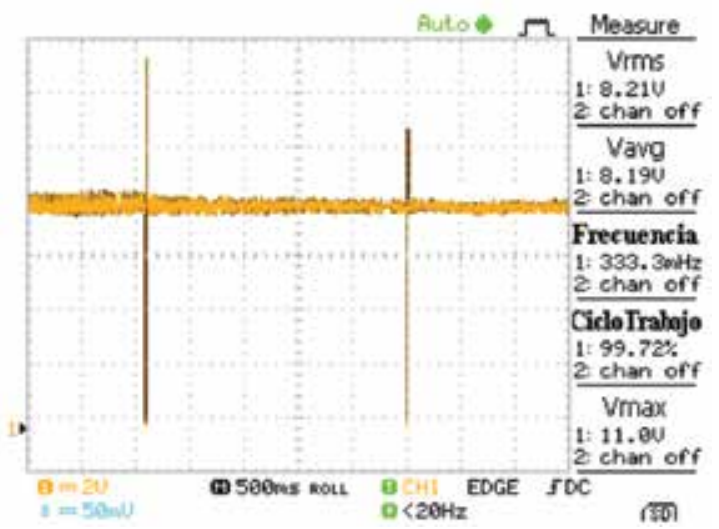

Figura 6. Regulación de tensión por SMC ante perturbaciones de carga (FPGA)

Fuente: los autores

De otro lado la, figura 7 permite visualizar resultados para el mismo caso anterior, esta vez bajo una acción de control del tipo óptimo conmutado. Se observa que la respuesta evidencia alteraciones visibles debido a las perturbaciones aplicadas. Por tanto, en comparación con la acción SMC, este tipo de control presenta un menor desempeño que amerita un análisis exhaustivo para determinar las razones que justifican este comportamiento bastante lejano de las predicciones teóricas.

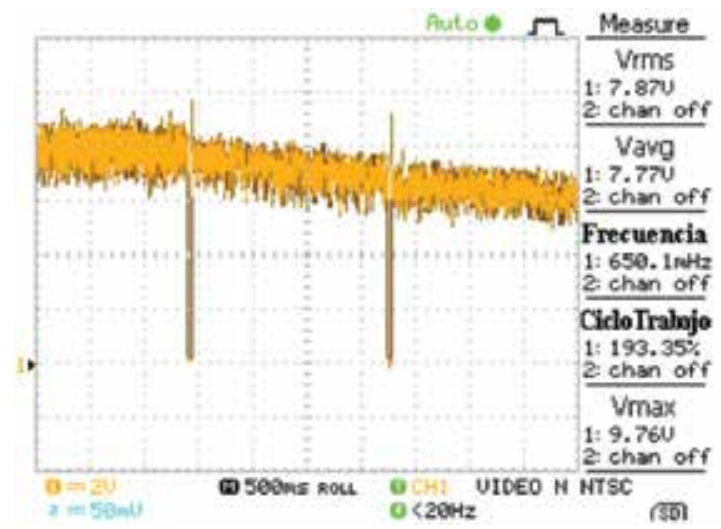

Figura 7. Regulación de tensión por control óptimo ante perturbaciones de carga (FPGA)

Fuente: los autores
La figura 8, por su parte, ilustra los resultados experimentales para la salida de voltaje en un circuito convertidor de potencia del tipo AC-DC (rectificador controlado) basado en un SCR, bajo la acción de perturbaciones en la carga cuando está sometido a un control del tipo proporcional (con ganancia $\mathrm{Kp}=32$ ) ejecutado en un FPAA. De la figura se evidencia un leve comportamiento transitorio sobreamortiguado que rápidamente se establece en un valor final diferente al deseado, es decir, se genera un error en estado estacionario.

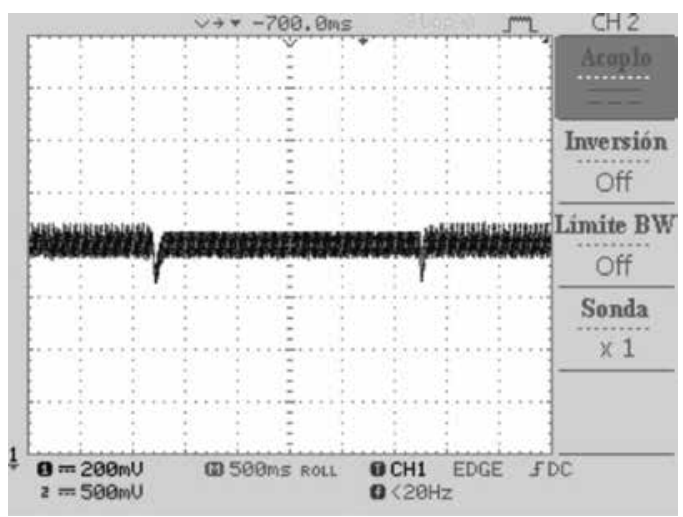

Figura 8. Regulación de tensión por control proporcional ante perturbaciones de carga (FPAA)

Fuente: los autores

Un resultado complementario a este último se presenta en la figura 9 para una acción de control PID sintonizado $(\mathrm{Kp}=6,4, \mathrm{Ki}=0,004, \mathrm{Kd}=0,004)$. Como se observa en la figura, el comportamiento transitorio es mucho más evidente respecto a la oscilación. Sin embargo, como era de esperarse, se corrige el error en estado estacionario gracias a la acción integral.

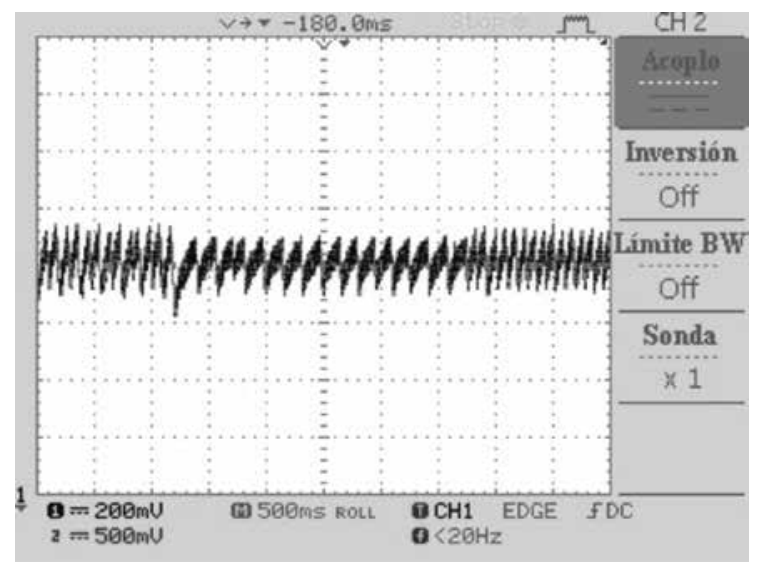

Figura 9. Regulación de tensión por SMC ante perturbaciones de carga (FPAA)

Fuente: los autores 
Finalmente, la figura 10 permite verificar la forma de onda obtenida tras ejecutar sobre un FPAA la acción de control smc. Nuevamente se observa una respuesta plana que anula de manera rápida el efecto de las perturbaciones, permitiendo que el voltaje de salida se establezca al valor deseado de manera casi inmediata.

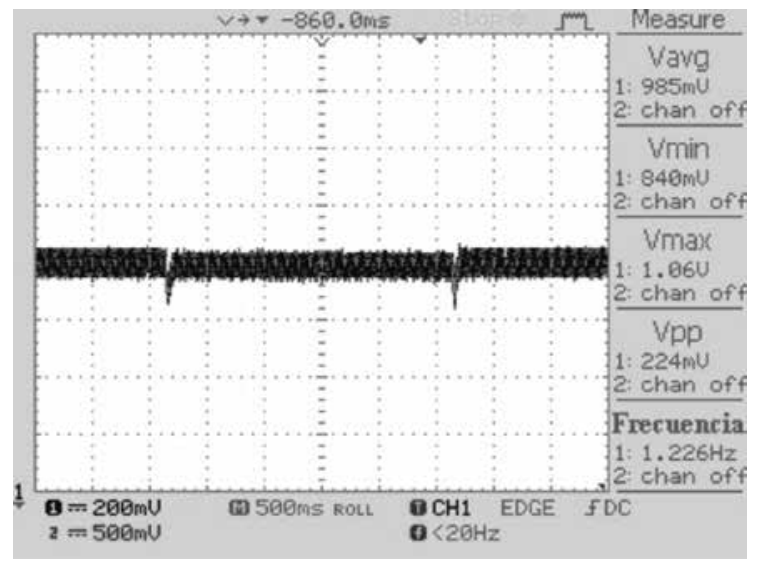

Figura 10. Regulación de tensión por SMC ante perturbaciones de carga (FPAA)

Fuente: los autores

Las configuraciones circuitales y los valores de parámetro empleados para obtener los resultados presentados se detallan en las referencias [17] y [18], respectivamente, para los casos de tecnologías FPGA y FPAA.

\section{Discusión}

De los resultados presentados en las figuras 6 y 10 , se confirma un apropiado desempeño en el comportamiento del circuito convertidor de potencia ante la acción de perturbaciones, para mantener regulado el voltaje en su salida cuando se aplica la técnica de control por modos deslizantes SMC. Este resultado es independiente de la plataforma de implementación utilizada, pues se verifica tanto para FPGA como para FPAA. También se evidencia una degradación en el comportamiento del control óptimo conmutado según resultados de la figura 7 , lo cual sugiere una revisión del método de implementación para determinar las consideraciones de tipo práctico que deben agregarse para asegurar su desempeño teóricamente "óptimo". Las técnicas de control convencional como P y PID demuestran un comportamiento dinámico inferior al obtenido por una técnica de control híbrido eficiente como pudo evidenciarse al comparar las figuras 8, 9 y 10.

\section{Conclusiones}

Se han presentado técnicas de control híbrido como solución potencial al problema de regulación de los niveles de tensión a la salida de circuitos convertidores de potencia, para el mejoramiento de la eficiencia energética. Los sistemas de ejemplo corresponden con un convertidor DC-DC tipo Buck y un convertidor ACDC tipo rectificador controlado basado en SCR. Se ha presentado una metodología para implementación de los algoritmos de control híbrido dividida en tres pasos: modelado matemático, análisis numérico e implementación física. Se han considerado como técnicas de ejemplo el control por modos deslizantes SMC y el control óptimo conmutado. Los mejores resultados en la práctica se obtuvieron para la técnica SMC. El trabajo complementario incluye la revisión de los aspectos prácticos para mejorar el desempeño del control óptimo y la aplicación de la metodología presentada en niveles de potencia mayores que permitan tener un impacto a escala industrial.

\section{Agradecimientos}

Los autores desean agradecer a la Universidad Industrial de Santander por financiar este trabajo a través del proyecto de investigación identificado con el código VIE-UIS 5568.

\section{Referencias}

[1] Z. Malekjamshidi y J. Mohammad, "A hybrid ANN and PID classic controller applied to a cascade LC resonant converter", Conference on Control, Instrumentation and Automation (ICCIA), 2nd International, Shiraz, Irán, pp. 675-681, 2011.

[2] C. Klumpner, "Hybrid power converters: An exploration of benefits", ISIE 2008. IEEE International Symposium on Industrial Electronics, 2008, pp. 1-27.

[3] K. Jezernik y R. Horvat, "FPGA Hybrid Controller for Unity Power Factor". Ohrid, Power Electronics and Motion Control Conference (EPE/PEMC), 2010, 14th International, T13-11-T13-16.

[4] A. Ghadam, S. Burglechner, A. H. Gokceoglu, M. Valkama y A. Springer, "Implementation and Performance of DSP-Oriented Feedforward Power Amplifier Linearizer", IEEE Transactions on Circuits and Systems I: Regular Papers, Vol. 59, No. 2, pp. 409-425, 2012. 
[5] A. Deese, y C. O. Nwankpa, Utilization of FPAA technology for emulation of multi-scale power system dynamics in smart grids, San Diego, CA: Power and Energy Society General Meeting, 2012, IEEE.

[6] F. Chierchie, y E. E. Paolini, "Discrete-time modeling and control of a synchronous buck converter". Argentine School of Micro-Nanoelectronics, Technology and Applications, EAмTA, Conferencia, Oct, 2009, pp. 5-10.

[7] I. Utkin, Sliding Modes and their applications in Variable Structure Systems, Moscú: Mir Publ.,1978

[8] D. E. Kirk, Optimal control theory: An introduction, Englewood Cliffs, NJ: Prentice-Hall, 1970.

[9] J.J.Slotine y W. Li, Applied Nonlinear Control, Englewood Cliffs, NJ: Prentice-Hall, 1991.

[10] Landau y Lifshitz, Mecánica, Barcelona: Ed. Reverté, 1991, pp.158-175.

[11] H. Goldstein, Mecánica clásica, Barcelona: Ed. Reverté, 1994.

[12] S. Boksuwan y T. Benjanarasuth, "Code generation of fractional filters for dsPIC microcontrollers”. Bali: TENCON 2011-2011 IEEE Region 10 Conference, 2011, pp. 1275-1279.
[13] A. Doboli, P. Kane y D. Van Ess, "Dynamic reconfiguration in a PSoC device" International Conference on Field-Programmable Technology, Sydney, Nsw, 2009, pp. 361-363.

[14] M. H. Rashid, Electrónica de potencia. Circuitos, dispositivos y aplicaciones, México: Prentice Hall Hispanoamericana S. A., 1993.

[15] L. G. Fuentes, Y. K. Tutira y P. J. Esteban, “Diseño y simulación de estrategias de control conmutado en convertidores de potencia", trabajo de grado, Bucaramanga: Universidad Industrial de Santander, 2012.

[16] D. G. Luenberger, Introduction to dynamic systems: Theory, Models and Applications, Nueva York: Wiley, 1979.

[17] M. F. Gómez, L. G. Navarro, D. F. Jácome y R. Alzate, "Estrategias de control conmutado aplicadas a circuitos convertidores de potencia”. XVIII Simposio de Tratamiento de Señales, Imágenes y Visión Artificial (sTsIvA), Bogotá, 2013.

[18] C. R. Durán, J. A. Flórez y R. Alzate, "Regulación de voltaje por control de disparo empleando tecnologías FPAA". Sometido a consideración del IV Simposio Internacional de Electrónica, Telecomunicaciones y Ciencias de la Computación ETCC, ITM, Medellín, 2013. 